\title{
RELAÇÕES ENTRE DESEMPENHO EM 200M NADO CRAWL E VARIÁVEIS CINÉTICAS DO TESTE DE NADO ESTACIONÁRIO*
}

\author{
DR. FLÁVIO ANTÔNIO DE SOUZA CASTRO \\ Doutor em ciências do movimento humano - Universidade Federal do Rio Grande do Sul (UFRGS) \\ Professor adjunto do Departamento de Educação Física (Rio Grande do Sul - Brasil) \\ E-mail: souza.castro@ufrgs.br
}

GRAD. THIAGO SILVA DE OLIVEIRA

Bacharel em educação física - UFRGS (Rio Grande do Sul - Brasil)

E-mail: thiagomib@yahoo.com.br

MS. FELIPE COLLARES MORÉ

Mestre em ciências do movimento humano - UFRGS

Sócio-proprietário da Even Faster Sports - Porto Alegre (Rio Grande do Sul - Brasil)

E-mail: fcmore@gmail.com

\section{DR. CARLOS BOLLI MOTA}

Doutor em ciências do movimento humano - Universidade Federal de Santa Maria (UFSM) Professor adjunto do Centro de Educação Física e Desportos - (Rio Grande do Sul - Brasil)

E-mail: bollimota@gmail.com

\section{RESUMO}

O objetivo deste estudo é relacionar o desempenho (DES) em 200m nado crawl e variáveis cinéticas do teste de nado estacionário (TNE): impulso total (IMP), taxa de variação de impulso (TIMP) e pico de força (PF). De 12 nadadores competitivos (idade média de 18,3 $\pm 2,9$ anos) foram obtidos IMP, TIMP, PF e DES em 200m nado crawl (este transformado em unidades pontuais). Com o desempenho (711,8 \pm 29, I pontos), o IMP (2663,6 \pm 150,6 $N \cdot s)$ apresentou correlação $(r=0,876 ; p<0,001)$, enquanto a $\operatorname{TIMP}(-24,45 \pm 14,38$ $N)$ e o PF $(217,48 \pm 29,10 N)$ não apresentaram correlações $(r=-0,553 ; p=0,062$ e $r=-0,19 ; p=0,7 / 4$; respectivamente). O impulso total obtido no TNE pode ser utilizado como parâmetro de avaliação da capacidade de força aplicada ao longo do tempo e como preditor do desempenho na prova de $200 \mathrm{~m}$ nado livre.

PALAVRAS-CHAVE: Natação; nado estacionário; impulso; cinética.

* Agradecimentos à Capes pela bolsa de doutorado sanduíche no exterior. Não houve conflitos de interesses para realização do presente estudo. 
A medição das forças geradas por um nadador e o arrasto que elas the oferecem têm sido alvo de muitos estudos (PAPOTI et al., 2003; TAYLOR et al., 2003; Dopsaj et al., 2003; SWAINE; Doyle, 1999; Kolmogorov; DuplischeVA, 1992; KESKINEN; KOMI, I993; YEATER et al., 198I), tendo em vista que a relação entre as forças resistivas e as forças propulsivas é que definem o deslocamento do nadador no meio aquático. Quando a força propulsiva for maior que o arrasto, o nado é realizado em aceleração positiva; quando há equilíbrio entre as forças de arrasto e de propulsão, é assumido que o nado deve ser realizado em velocidade constante; já quando o arrasto é maior que a força propulsiva, o nado é realizado em aceleração negativa (TousSAINT; BEEK, 1992). Akis e Orcan (2004) afirmam que, no estudo da biomecânica da natação, um objetivo fundamental é determinar a força propulsiva desenvolvida pelo nadador, o arrasto a seu deslocamento e suas relações com a técnica e o desempenho do nadador.

Uma das técnicas que é amplamente utilizada, cujos resultados estão relacionados à força propulsiva, é o chamado tethered swimming (teste de nado estacionário - TNE). A fim de avaliar a resposta das forças aplicadas pelo nadador na água, o TNE utiliza transdutores de força que mensuram as forças resultantes daquelas aplicadas pelo nadador, a partir de um cabo fixado ao nadador, a um transdutor de força, à borda da piscina e a um computador (KESKINEN; KOMI, 1993).

Dopsaj et al. (2003) afirmam que o TNE é o teste mais utilizado para medir a força realizada na natação. Nadar, segundo os autores, consiste em uma série de movimentos cíclicos realizados alternadamente por pernas e braços, e cada ciclo resulta em uma força característica que impulsiona o indivíduo para frente, possível pela contração dos músculos envolvidos. Quando mensurada pela técnica de TNE, essa força é definida como uma força propulsiva e é apresentada como uma sinusoide ao longo do tempo, que descreve seu comportamento temporal. Os autores afirmam, ainda, que a força mensurada pelo TNE e a velocidade de nado variam de acordo com a idade maturacional, o nível competitivo do indivíduo e a distância avaliada.

Exemplos da utilização TNE podem ser encontrados nos estudos de Costill (1985) e Papoti et al. (2003). Costill (1985) encontrou correlação de 0,84 entre velocidade de nado (obtida da distância de 25 jardas) e força mensurada em TNE entre 76 nadadores universitários de ambos os sexos. Já Papoti et al. (2003), com o objetivo de padronizar um protocolo específico para avaliar a aptidão anaeróbia de nadadores e verificar suas correlações com o desempenho nas provas de $200 \mathrm{~m}$ e $400 \mathrm{~m}$ nado livre, avaliaram 13 nadadores competitivos entre 15 e 18 anos com o 
método de TNE. Dentre os resultados, destacaram-se correlações entre o impulso (integral entre força aplicada e mensurada pela TNE e tempo do teste, 30 segundos) e o desempenho em 200m e 400m nado crawl, todos testes de esforço máximo. Os autores concluíram que o impulso, obtido com TNE, é efetivo na predição de performance nas provas de $200 \mathrm{~m}$ e $400 \mathrm{~m}$ realizadas em nado crawl.

OTNE permite verificar o IMP produzido pelo nadador que, segundo Castro (2007), é a integral entre a força e o tempo, expresso em Ns, o PF (força máxima obtida durante o teste, expressa em N) e a taxa de variação de impulso (TIMP, variação do impulso a cada 5 segundos, expressa em N). Os estudos realizados com TNE (PAPOTI et al., 2003; TAYLOR et al., 2003) têm verificado a relação entre impulso total e desempenho, mas não se sabe se há alguma relação entre o pico de força (PF) bem como a taxa de variação de impulso (TIMP) com o desempenho nos 200m. Resultados prévios demonstram correlações de PF com desempenho em provas mais curtas, como os 50m e os 100m nado livre (RISCH; CASTRO, 2007; MORÉ et al., 2005), que apresentam maior dependência da força e da potência muscular que a prova de $200 \mathrm{~m}$ nado livre. As provas de natação em piscina variam de $50 \mathrm{~m}$ a $1500 \mathrm{~m}$ nos estilos livre, costas, peito e borboleta (estes três apenas nas distâncias de $50 \mathrm{~m}$ a $200 \mathrm{~m}$ ). A prova de $200 \mathrm{~m}$ nado livre, devido às suas características metabólicas com grande participação do sistema glicolítico que estaria relacionado a altos níveis de fadiga, apresenta uma condição de desafio tanto para o técnico quanto para o nadador. Em relação à contribuição energética, Gastin (200 I) indica que esforços máximos, independente do exercício, de aproximadamente 120 segundos de duração, apresentam participação de 37\% de metabolismo glicolítico e de 63\% de metabolismo aeróbio. Já Capelli et al. (1998) encontraram, em esforços máximos de $183 \mathrm{~m}$ (200 jardas) em nado crawl, que duraram em média I I 2 segundos, 39\% de contribuição anaeróbia e $61 \%$ de contribuição aeróbia.

A TIMP estaria relacionada à capacidade de aplicação de força ao longo do tempo e à fadiga. Segundo Castro (2007), a curva representativa do comportamento do impulso, em nado estacionário, com redução significativa dos valores ao longo do tempo, apresenta grande similaridade com as curvas obtidas de testes de Wingate, que permitem avaliação das potências e capacidades anaeróbias. Tendo em vista essa possível relação entre TIMP e capacidades fisiológicas do nadador, torna-se interessante verificar a relação entre TIMP e desempenho na prova de 200m nado livre. Hawley e Williams ( 199 | ) encontraram correlação entre potência anaeróbia de membros superiores (obtida de teste de Wingate) e desempenho nos $50 \mathrm{~m}$ de natação. Os valores de correlação relatados por Hawley e Williams ( 99 |) foram decrescendo até a distância de $400 \mathrm{~m}$, mostrando uma menor importância da potência de membros superiores quando as provas são realizadas sob menores 
velocidades de nado. Embora Guglielmo e Denadai (2000) não tenham encontrado correlações entre os parâmetros do teste de Wingate para membros superiores e o desempenho em natação entre as distâncias de 14m, 25m, 50m e 400 m, há que se considerar a especificidade do gesto e do meio de realização dos testes para a avaliação da capacidade anaeróbia: enquanto o teste de Wingate adaptado a membros superiores é realizado em cicloergômetro e no seco, o TNE é realizado em natação e no próprio meio aquático.

Já o PF estaria relacionado à máxima capacidade de produção de força do indivíduo e, portanto, poderia apresentar alguma relação com o desempenho, visto que o sujeito que aplica a maior força poderia, teoricamente, aplicar mais força durante o nado e, assim, apresentaria um melhor desempenho. Risch e Castro (2007) avaliaram nove nadadores de nível universitário a fim de verificar a correlação dos dados obtidos (PF, desempenho em 50m e variáveis antropométricas). Os autores encontraram correlações significativas entre PF e desempenho, estatura e massa, e entre desempenho e massa. Moré et al. (2005) avaliaram seis nadadores e quatro triatletas e encontraram correlação significativa entre força aplicada por braçada e desempenho em $100 \mathrm{~m}$ para nadadores, mas não encontraram correlação significativa desses parâmetros entre os triatletas. Embora o TNE seja utilizado a fim de mensurar as forças durante o nado, o arrasto, componente fundamental em relação ao desempenho, é negligenciado no método, já que o nadador fica estacionário.

Considerando os resultados prévios encontrados com o TNE, as características energéticas da prova de $200 \mathrm{~m}$ nado livre e as possibilidades de análise das informações advindas do TNE, este trabalho tem como objetivo verificar as relações entre o desempenho em 200m nado crawl com parâmetros do teste de nado estacionário (impulso total, taxa de variação de impulso e pico de força).

\section{MATERIAL E MÉTODOS}

A população deste estudo foi de nadadores competitivos com, no mínimo, 16 anos de idade e quatro anos de experiência na modalidade, com índice de participação em Campeonato Brasileiro de Natação. O tamanho amostral ( 12 nadadores) foi definido após aplicação da equação que determina o erro de medida para as principais variáveis em análise. Os participantes deste estudo foram devidamente informados sobre todos os procedimentos realizados (um Termo de Consentimento foi apresentado aos participantes). Este estudo foi aprovado pelo Comitê de Ética em Pesquisa da universidade onde foi realizado (processo n. 200769l). 
Foram utilizados os seguintes instrumentos de avaliação:

I. Variáveis antropométricas: balança marca Filizola, modelo 31, com resolução de 0,I kg; estadiômetro marca Filizola, modelo 3 I , com resolução de $0,01 \mathrm{~m}$, trena com resolução de $0,01 \mathrm{~m}$.

2. Variáveis cinéticas: microcomputador portátil (HP, processador Pentium II, 20 gigabytes de memória, 128 megabytes de memória RAM); célula de carga modelo ZX 250 (Alfa Instrumentos, SP) com sensibilidade de $2 \mathrm{mVN}$ e capacidade para $250 \mathrm{~kg}$; condicionador de sinais 3103 (Alfa Instrumentos, SP); placa conversora analógica digital PCM-DASI65//6 (Computer Boards, EUA) de 16 bytes e 300 kHz; sistema de aquisição de dados SAD32p (Laboratório de Medições Mecânicas, Universidade Federal do Rio Grande do Sul); cabo de aço com 6m de comprimento e $3 \mathrm{~mm}$ de diâmetro e um cinto de tecido preso a uma de suas extremidades; ficha para apontamento dos dados.

3. Variáveis cinemáticas: dois cronômetros manuais (Casio, HS-50W, resolução de I/l00).

Todos os protocolos de avaliação foram aplicados em piscina de $25 \mathrm{~m}$, aquecida e coberta, com água entre $29^{\circ} \mathrm{C}$ e $30^{\circ} \mathrm{C}$. Os nadadores realizaram aquecimento, nadando $800 \mathrm{~m}$ nado livre, em baixa intensidade. Em seguida, realizaram um teste máximo de $200 \mathrm{~m}$, no estilo crawl. Após permaneceram I 5 minutos em repouso passivo, executaram $600 \mathrm{~m}$ nadando em baixa velocidade, caracterizando um nado regenerativo. Tais procedimentos foram realizados a fim de evitar possíveis efeitos do esforço prévio sobre o TNE. O tempo total de repouso (passivo mais ativo) foi de cerca de 30 minutos, suficiente para a recuperação adequada para um novo esforço máximo (WHITEBREAD et al., 2005). Em seguida os nadadores foram familiarizados com o TNE, que foi realizado em 35 segundos de nado crawl, em baixa intensidade. Depois, realizaram o TNE, que, conforme Fomitchenko (1999), consiste em 35 segundos executando o nado crawl na maior intensidade possível.

Os dados obtidos das coletas necessitaram de diferentes processamentos até a obtenção do valor final. Os protocolos para obtenção e processamento das variáveis foram:

I. Variáveis antropométricas: características físicas dos participantes foram obtidas anteriormente à realização dos protocolos citados. A massa corporal foi obtida quando o participante, trajando apenas sunga de natação, permaneceu em pé, com um mínimo de oscilações corporais, de frente 
para o avaliador e para a régua de marcação, sobre a balança. A estatura foi obtida quando o participante permaneceu em pé, posicionado sobre a balança e de costas para a régua, utilizando-se o estadiômetro da balança. A envergadura foi mensurada com o participante na posição de decúbito dorsal, sobre um colchonete de $3 \mathrm{~cm}$ de espessura, no solo, ombros abduzidos a $90^{\circ}$, cotovelos, punhos e dedos em extensão, sobre o solo. A máxima distância entre as extremidades das mãos (terceiro dedo) foi considerada a envergadura.

2. Variáveis cinemáticas: além da obtenção do tempo total dos $200 \mathrm{~m}$, transformado em unidades pontuais (considerando o recorde mundial na época da coleta como 1000 pontos e aplicando-se uma regra de três para os tempos individuais obtidos). De modo complementar, foram anotados os tempos a cada $50 \mathrm{~m}$ e a frequência de ciclos de cada $25 \mathrm{~m}$, com a anotação do tempo necessário para a realização de três ciclos completos entre os $10 \mathrm{~m}$ e os $20 \mathrm{~m}$ de cada percurso e o cálculo do quociente entre 3 (ciclos) e o tempo (em segundos), expressando a frequência de ciclos em Hz. Ao longo do TNE foi registrado ainda o número de ciclos executados nos 30 segundos intermediários do teste, o que permitiu a identificação da frequência de ciclos do TNE.

3. Variáveis cinéticas: foram obtidas com a utilização do TNE, o qual consiste da célula de carga fixada à cintura do nadador e à borda da piscina. A célula de carga foi conectada ao indicador de sinais e este, ao computador. Durante o TNE, o sinal de força foi coletado a uma taxa de amostragem de $500 \mathrm{~Hz}$ no software SAD32p. Previamente a cada coleta o equipamento foi calibrado, com a utilização de massas conhecidas.

Depois de obtido, o sinal de força foi filtrado (filtro média-móvel com frequência de corte de $15 \mathrm{~Hz}$ ). Foram desprezados os primeiros e últimos 2,5 segundos do teste. No mesmo programa (SAD32p), pela aplicação de uma integral (Simpson), foi identificado o impulso total (IMP) obtido por cada nadador, durante os 30 segundos intermediários do teste. Além do IMP, foi calculada a taxa de variação do impulso (TIMP), com o recorte de seis janelas de 5 segundos de duração, ao longo dos 30 segundos do sinal de força e identificado o impulso médio de cada janela. Foi aplicada, então, uma regressão linear entre o impulso médio de cada janela e o tempo, regressão esta que forneceu uma equação linear para cada indivíduo. Dessas equações de regressão linear, o coeficiente angular foi considerado o TIMP. O PF foi identificado na curva de força, após filtragem, como o valor máximo registrado ao longo do teste. 
A análise estatística contou com cálculo das médias, desvios e erros padrão. Foi verificada a normalidade dos dados (Shapiro-Wilk). O coeficiente de determinação $\left(r^{2}\right)$ foi calculado juntamente com as equações de regressão linear entre os valores de IMP a cada janela de 58 segundos e o tempo correspondente. Correlações entre impulso total e desempenho, taxa de variação de impulso e desempenho, pico de força e desempenho foram testadas com o Teste de correlação linear produtomomento de Pearson. Comparações entre as frequências de ciclos foram realizadas com um teste t de Student para dados pareados. Já a duração dos primeiros $50 \mathrm{~m}$ do teste de $200 \mathrm{~m}$ foi comparada aos 30 segundos do TNE por meio de um teste t de Student simples. Considerou-se $\alpha<0,05$. Utilizou-se o programa SPSS v. I5.0 para as análises.

\section{RESULTADOS E DISCUSSÃO}

As características dos participantes deste estudo são apresentadas na tabela I .

Tabela I: Características dos participantes da amostra, $\mathrm{n}=12$, em média \pm desvio-padrão (d.p.)

\begin{tabular}{lllll}
\hline & Idade & Massa & $\begin{array}{l}\text { Estatura } \\
(\mathrm{cm})\end{array}$ & Envergadura $(\mathrm{cm})$ \\
\hline Média & 18,3 & $(\mathrm{~kg})$ & 174,25 & 181,04 \\
d.p. & 2,9 & 66,36 & 5,75 & 7,47 \\
\hline
\end{tabular}

A figura I apresenta o resultado do teste de nado estacionário característico de um indivíduo da amostra após filtragem.

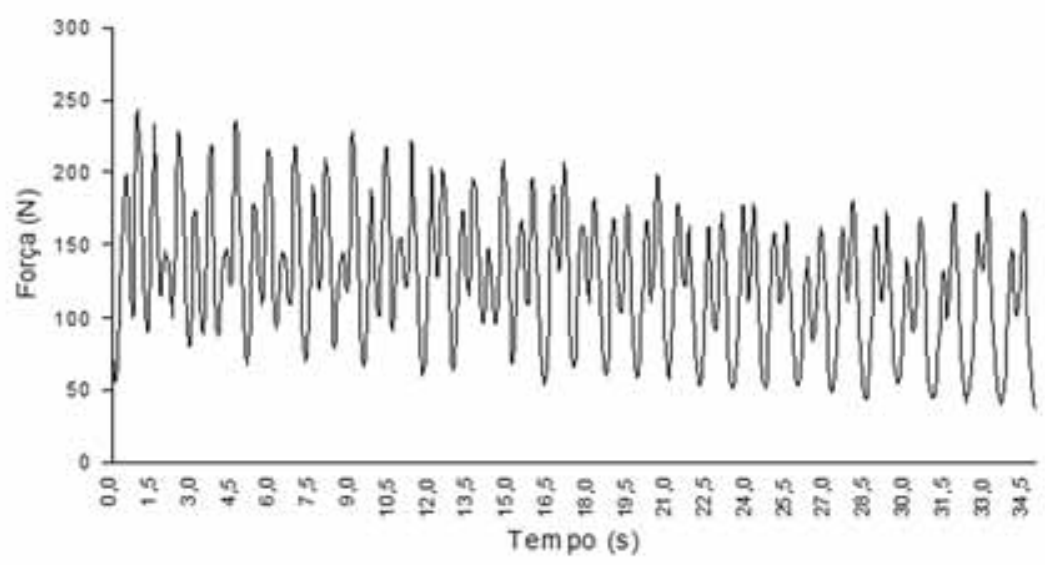

Figura I: Curva de força obtida do TNE, após filtragem, de um indivíduo participante da amostra. 
A figura 2 apresenta o valor médio e os desvios padrão do impulso para cada janela de 5 segundos do TNE e o coeficiente de determinação entre o tempo e o impulso.

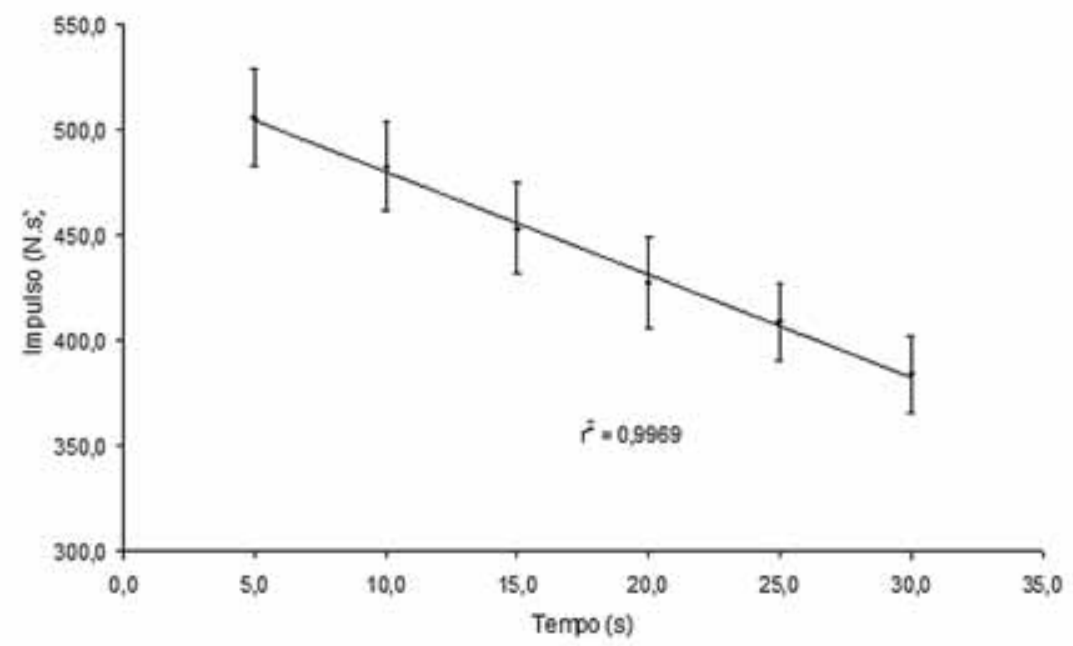

Figura 2: Comportamento do impulso, em média \pm desvio padrão a cada janela de 5 segundos do TNE e coeficiente de correlação entre IMP e tempo, $n=12$.

Os resultados de desempenho, impulso total, taxa de variação de impulso e pico de força são apresentados na tabela 2.

Tabela 2: Resultados de desempenho, em média \pm desvio padrão (d.p.) em tempo (DEST) em pontos (DES), tempo dos primeiros 50m (T50), impulso total (IT), taxa de impulso (TIMP) e pico de força (PF), $\mathrm{n}=12$

\begin{tabular}{ccccccc}
\hline & DEST & DES & T50 & IMP & TIMP & PF \\
& $(\mathrm{s})$ & $($ pontos $)$ & $(\mathrm{s})$ & $(\mathrm{Ns})$ & $(\mathrm{N})$ & $(\mathrm{N})$ \\
\hline Média & 130,17 & 711,80 & 30,2 & 2663,66 & $-24,45$ & 217,48 \\
d.p. & 2,9 & 29,10 & 0,89 & 150,6 & 14,38 & 29,10 \\
\hline
\end{tabular}

Os valores de impulso total, obtidos pelos nadadores, foram positiva e significativamente correlacionados $(r=0,876 ; p<0,00$ I) com o desempenho. A figura 3 apresenta a dispersão entre o impulso total e o desempenho. 


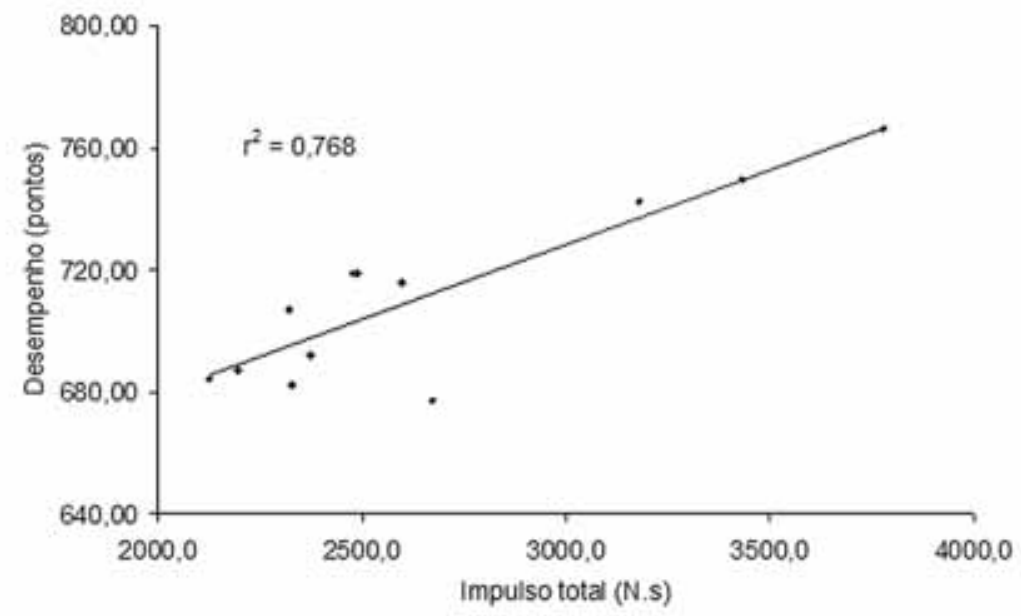

Figura 3: Dispersão entre impulso total e desempenho, com valor do coeficiente de determinação, $\mathrm{n}=12$.

Os valores de taxa de variação de impulso não apresentaram correlação significativa com o desempenho $(r=-0,553 ; p=0,062)$. A figura 4 apresenta a dispersão entre taxa de impulso e desempenho.

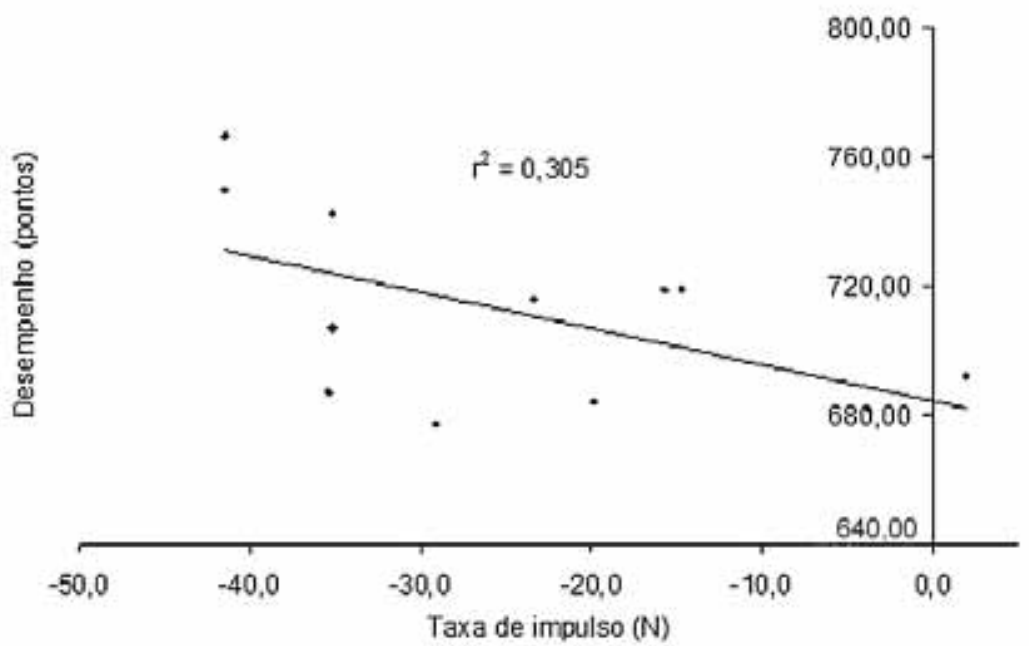

Figura 4: Dispersão entre taxa de impulso e desempenho com valor de coeficiente de determinação, $\mathrm{n}=12$. 
O pico de força não apresentou correlação significativa com o desempenho $(r=-0,19 ; p=0,7 \mid 4)$. A figura 5 apresenta a dispersão entre o pico de impulso e o desempenho.

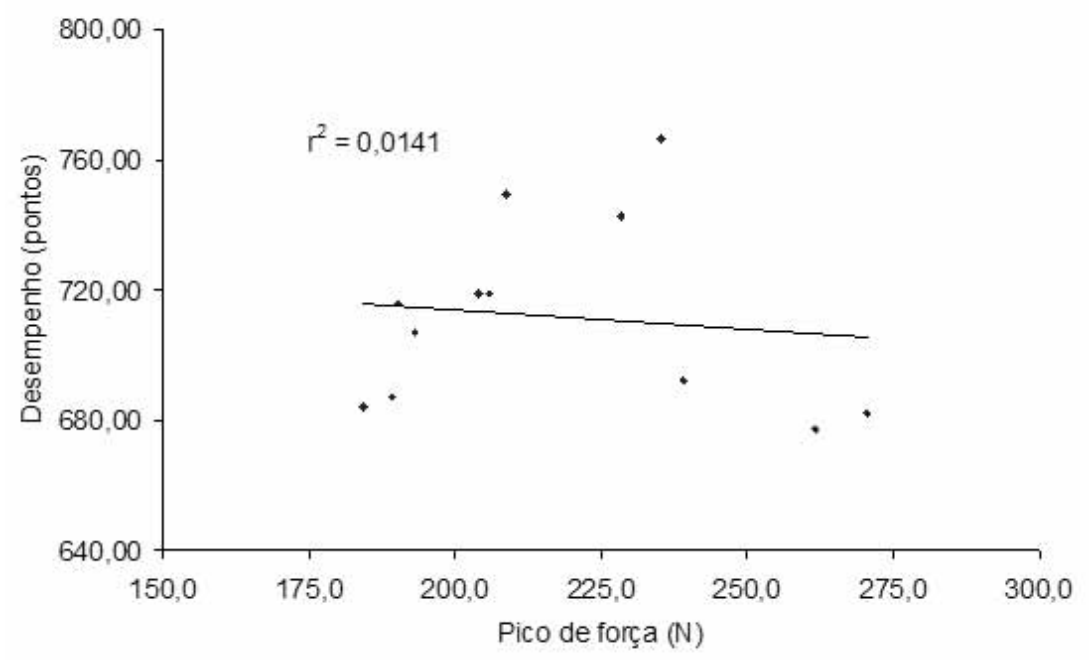

Figura 5: Dispersão entre pico de impulso e desempenho com valor de coeficiente de determinação, $\mathrm{n}=12$.

A tabela 3 apresenta os valores médios de frequência de ciclos de braçadas, em média \pm desvio padrão (d.p.) para os 200m (FC200), para os primeiros $50 \mathrm{~m}$ dos 200m (FC50) e para o TNE (FCTNE).

Tabela 3: Resultados de frequência de ciclos, em média \pm desvio padrão (d.p.) em Hz dos 200m (FC200), para os primeiros 50m (FC50) e do TNE (FCTNE), $\mathrm{n}=12$.

\begin{tabular}{cccc}
\hline & $\mathrm{FC} 200(\mathrm{~Hz})$ & $\mathrm{FC} 50(\mathrm{~Hz})$ & $\mathrm{FCTNE}(\mathrm{Hz})$ \\
\hline Média & 0,69 & 0,74 & 0,73 \\
d.p. & 0,07 & 0,08 & 0,07 \\
\hline
\end{tabular}

O presente estudo apresentou como objetivo verificar as relações entre o desempenho na prova de $200 \mathrm{~m}$ livre e parâmetros do teste de nado estacionário (IMP, TIMP e PF). Estudos realizados com o TNE já haviam observado a relação entre IMP e desempenho, mas não foram encontrados estudos, até então, que mostrassem se havia alguma relação entre o PF, bem como a TIMP, com o desempenho em 200m. Este estudo teve como hipótese que tanto a TIMP quanto o PF apresentariam correlação com o desempenho, já que a TIMP estaria relacionada à 
capacidade de manutenção dos esforços pelo nadador e que o PF estaria relacionado à máxima capacidade de produção de força do indivíduo. Os resultados mostraram correlação significativa entre o desempenho e o impulso total, e não mostraram correlação estatisticamente significativa entre desempenho e taxa de variação de impulso, nem entre desempenho e pico de força.

A curva representativa do comportamento do impulso, com redução significativa dos valores (Figura 2) de 506,2 $\pm 23,2$ Ns, no primeiro trecho de 5 segundos, para 384,0 \pm । 8, I Ns, no último trecho de 5 segundos, apresenta grande similaridade às curvas de potência obtidas de testes de Wingate, que permitem avaliação das potências e capacidades anaeróbias (LAURENT JR. et al., 2007). De acordo com Laurent Jr. et al. (2007), o teste anaeróbio de 30 segundos de Wingate é o protocolo mais empregado para mensuração de respostas anaeróbias. Esse teste envolve uma realização máxima de exercício por 30 segundos em um ciclo ergômetro, que permite avaliar pico de potência, potência média e índice de fadiga. Uma resposta típica de um teste de Wingate é um rápido incremento na potência produzida, atingindo um pico nos primeiros segundos de execução e, depois, uma incapacidade de manutenção da potência, com um declínio exponencial de potência produzida (LAURENT JR. et al., 2007) ao longo do teste, similar ao TNE (Figura 2).

Estabelecendo um paralelo com um teste de Wingate, os valores de impulso obtidos nos segundos iniciais (pico de impulso) seriam representativos das potências anaeróbias (alática e lática) e o comportamento dos valores subsequentes estaria relacionado às capacidades anaeróbias alática e lática (LAURENT JR. et al., 2007). No presente estudo, utilizou-se o impulso produzido ao longo do TNE. Os valores foram obtidos pela integral da força produzida pelo nadador dentro da água e o tempo decorrido. Como o tempo é o mesmo para todos os indivíduos, variações de impulso só podem ocorrer pela variação de força intra e interindivíduos.

No entanto, entre nadadores, é mais comum a avaliação da sua capacidade anaeróbica com a utilização do teste de Wingate para membros inferiores ou superiores (COLANTONIO et al., 2003). A realização de testes de Wingate entre nadadores, embora possibilite a avaliação das capacidades anaeróbicas, pode não representar a especificidade de teste que o gesto desportivo exige. Nesse caso, a utilização de um protocolo em natação, mesmo que estacionária (com todas as limitações já apresentadas), parece ser mais eficiente para essa avaliação do que o teste de Wingate, realizado fora da água, com ações musculares menos específicas.

Sobre a especificidade da avaliação em nado estacionário, a comparação entre as frequências médias de ciclos de braçadas dos 200m (FC200 = 0,69 \pm 
$0,07 \mathrm{~Hz}$, Tabela 3) e do TNE (FCTNE $=0,73 \pm 0,07 \mathrm{~Hz}$, Tabela 3) não mostrou diferença. De modo mais específico, compararam-se os valores de FCTNE com os valores de $\mathrm{FC}$ dos primeiros $50 \mathrm{~m}$ do teste de $200 \mathrm{~m}(0,74 \pm 0,08 \mathrm{~Hz})$. Os primeiros $50 \mathrm{~m}$ do teste de $200 \mathrm{~m}$ foram percorridos em 30,2 $\pm 0,89$ segundos, tempo similar à duração do TNE. Da mesma maneira, nenhuma diferença foi evidenciada. Essas similaridades entre as frequências de ciclo reforçam a possível utilização do teste de nado estacionário de 30 segundos como alternativa ao teste de Wingate para a avaliação das capacidades anaeróbias de nadadores. Cabe ressaltar que não era objetivo do presente estudo verificar medidas fisiológicas associadas ao TNE.

$O$ resultado do impulso total obtido pelos nadadores nos 30 segundos do TNE deste estudo (Tabela 2) foi similar ao valor de impulso encontrado por Papoti et al. (2003), que, avaliando 13 nadadores competitivos, entre 15 e 18 anos, em duas séries de 30 segundos de nado estacionário, com 20 minutos de intervalo passivo, encontraram valores de 25 I 0,2 \pm 105,0 Ns e 254 I,9 \pm | I 5,2 Ns, respectivamente, para a primeira e a segunda repetição de 30 segundos. Os valores de impulso, levemente mais altos, encontrados no presente estudo, podem estar relacionados tanto às características de idade dos participantes de ambos os estudos - já que, no presente estudo, as idades variaram entre 16 e 25 anos, com média de 18, $3 \pm 2,9$ anos (Tabela I), maior que o maior valor de idade do estudo de Papoti et al. (2003) - quanto à diferença de capacidade técnica, com nadadores mais experientes devendo apresentar melhor técnica e, consequentemente, maior capacidade de aplicação de força. Os do presente estudo apresentavam, no mínimo, quatro anos de experiência competitiva, contra dois do estudo citado.

Risch e Castro (2007), em estudo que avaliou nove nadadores, encontraram correlação significativa entre PF e desempenho nos 50m nado crawl, identificado pelo tempo na prova $(-0,67)$. O mesmo não ocorreu no presente estudo. É possível que essa diferença entre os resultados tenha ocorrido em razão do nível técnico dos nadadores, que no estudo de Risch e Castro (2007) eram de nível universitário, ao passo que no presente estudo são de nível nacional. Outra diferença foi a prova de referência. Enquanto no presente estudo foi estudada a prova de $200 \mathrm{~m}$ livre, Risch e Castro (2007) investigaram a prova de 50m, que apresenta uma característica fisiológica diferente, mais dependente da força e da potência muscular. Moré et al. (2005) encontraram correlação significativa entre força aplicada por braçada e desempenho, quando avaliaram seis nadadores de nível nacional. Esses nadadores possuíam nível técnico semelhante ao do presente estudo. A diferença é que, no estudo de Moré et al. (2005), a correlação testada foi entre o PF e o desempenho 
na prova de $100 \mathrm{~m}$ livre, cujas características tendem a ser intermediárias entre as provas de $50 \mathrm{~m}$ e de $200 \mathrm{~m}$ nado livre.

Keskinen e Komi ( 1993 ) realizaram um estudo que apresentava como objetivo comparar o desempenho técnico de nadadores competitivos com base na variação intracíclica de força, da velocidade e da potência durante o nado crawl executado sob máxima intensidade, em distância curta $(25 \mathrm{~m})$. Foi utilizando o método de TNE, quando foram avaliados 12 nadadores competitivos (idade média de 19,4 $\pm 3,4$ anos). Os autores concluíram que a potência pode ser utilizada como um parâmetro para se avaliar o desempenho técnico de nadadores, mas que o mesmo não ocorre com o parâmetro pico de força. $\bigcirc$ achado dos autores é semelhante ao deste estudo, que mostrou não haver correlação significativa entre desempenho e pico de força. Keskinen e Komi ( 1993) afirmam que os nadadores mais velozes não apresentaram os maiores picos de força. Desse modo, podemos afirmar que o pico de força não é um bom parâmetro para se utilizar quando da avaliação do desempenho em natação.

Quanto à TIMP, é possível afirmar, também, que não pode ser utilizada como parâmetro para se avaliar o desempenho, visto que não foi encontrada correlação significativa entre TIMP e desempenho. Castro (2007) afirma que o comportamento da curva de impulso (tendo como base a TIMP, Figura I) é semelhante ao comportamento da curva do teste de Wingate (potência em cicloergômetro) e faz um paralelo entre os dois testes (TNE e Wingate), sugerindo que o TNE, pela especificidade do teste, poderia apresentar melhores resultados de capacidade anaeróbia para nadadores. $\bigcirc$ presente estudo não foi capaz de relacionar PF nem TIMP com desempenho. Mais estudos deveriam ser realizados, com controle simultâneo de variáveis fisiológicas, a fim de se entender melhor a aplicabilidade desses parâmetros.

\section{CONCLUSÕES}

impulso total obtido do teste de nado estacionário pode ser utilizado como um parâmetro para se predizer o desempenho na prova de $200 \mathrm{~m}$ nado livre e a capacidade de aplicação de força ao longo do tempo, visto que apresenta correlação com o desempenho na prova. Já os parâmetros taxa de impulso e pico de força não se correlacionaram com o desempenho, e, portanto, não deveriam ser utilizados com o objetivo de predizer a capacidade de desempenho na prova de $200 m$ nado livre. 


\section{Relationship between $200 \mathrm{~m}$ front crawl stroke performance and tethered swimming test kinetics variables}

ABSTRACT: The purpose of this study is to relate $200 \mathrm{~m}$ front crawl performance (DES) and kinetics variables obtained from a tethered swimming test (TNE): total impulse (IMP), impulse rate (TIMP) and force peak (PF). From 12 competitive swimmers (mean age: $18.3 \pm 2.9$ years) were obtained IMP, TIMP, PF and DES in $200 \mathrm{~m}$ front crawl (this last transformed into unit points). Regarding to DES (7II.8 \pm 29. I points): IMP (2663.6 \pm 150.6 N-s) was significantly correlated $(r=0.876$; $p<0.001)$, whereas TIMP $(-24.45$

$\pm 14.38 \mathrm{~N})$ and PF $(217.48 \pm 29.1 \mathrm{~N})$ were not correlated $(r=-0.553 ; p=0.062$ and $r=-0.19 ; p=0.714$, respectively). Total impulse obtained from TNE can be used a force capacity along time assessment parameter and as a performance parameter predictor for the $200 \mathrm{~m}$ freestyle event.

KEY WORDS: Swimming; tethered swimming; impulse; kinetics.

\section{Relación entre desempeño en los $200 \mathrm{~m}$ nado crol y variables cinéticas del test de nado estacionario}

RESUMEN: O objectivo del estudo es relacionar el desempeño (DES) en 200m nado crol y variables cinéticas del test de nado estacionario (TNE): impulso total (IMP), tasa de variación de impulso (TIMP) y pico de fuerza (PF). De 12 nadadores competitivos (edad: 18,3 \pm 2,9 anos) se obtuvieron IMP, TIMP, PF y DES en 200 m nado crol (éste convertido en unidades puntuales). Con el desempeño (7II,8 \pm 29, I puntos): IMP (2663,6 \pm 150,6 $\mathrm{N} \cdot \mathrm{s})$ presentó correlación $(r=0,876 ; p<0,001)$, mientras $\operatorname{TIMP}(-24,45 \pm 14,38 \mathrm{~N})$ y PF $(217,48 \pm 29,10 \mathrm{~N})$ no presentaron correlaciones $(r=-0,553 ; p=0,062$ y $r=$ $-0,19 ; p=0,7$ I $;$; respectivamente). El impulso total obtenido en el TNE se puede utilizar como parámetro de evaluación de la capacidad de fuerza aplicada a lo largo del tiempo y como predictor del desempeño en la prueba de 200 m nado libre.

PALABRAS CLAVES: Natación; nado estacionario; impulso; cinética.

\section{REFERÊNCIAS}

AKIS, T.; ORCAN, Y. Experimental and analytical investigation of the mechanics of crawl stroke swimming. Mechanics Research Communications,Amsterdam, v. 31, p. 243-26I, 2004.

CAPELLI, C.; PENDERGAST, D.; TERMIN, B. Energetics of swimming at maximal speed in humans. European Journal of Applied Physiology, Heidelberg, v. 78, p. 385-93, 1998.

CASTRO, F. A. S. Determinantes do desempenho para a prova de 200m nado livre. 2007. I 52 f. Tese (Doutorado em Educação Física) - Escola de Educação Física, UFRGS, Porto Alegre, 2007. 
COLANTONIO, E. et al. Oxygen uptake during Wingate tests for arms and legs in swimmers and water polo players. Revista Brasileira de Medicina do Esporte, São Paulo, v. 9, p. I 4 I -44, mar. 2003.

COSTILL, D. L. Practical problems in exercise physiology research. Research Quarterly, Reston, v. 56, n. 4, p. 378-384, 1985.

DOPSAJ, M.; MATKOVI, I.; ZDRAVKOVI, I. The relationships between 50m - freestyle results and characteristics of tethered forces in male sprint swimmers: a new approach to tethered swimming test. Facta Universitatis. Physical Education and Sports, Nissa, v. I, n. 7 , p. 15-20, 2003.

FOMITCHENKO, T. G. Relationship between sprint speed and power capacity in different groups of swimmers. In: KESKINEN, K.; KOMI, P; HOLLANDER, A. (eds.) Biomechanics and medicine in swimming VIII. Jyväskylä: Department of Biology of Physical Activity, University of Jyväskylä, p. 209-211, 1999.

GASTIN, P. Energy system interaction and relative contribution during maximal exercise. Sports Medicine, Indianapolis, v. 3 I, n. 10, p. 725-74I, 2001.

GUGLIELMO, L. G. A.; DENADAI, B. S. Assessment of anaerobic power of swimmers: the correlation of laboratory tests on an arm ergometer with field tests in a swimming pool. The Journal of Strength and Conditioning Research, Philadelphia, v. 4, p. 395-398, 2000.

HAWLEY, J. A.; WILLIAMS, M. M. Relationship between upper body anaerobic power and freestyle swimming performance. International Journal of Sports Medicine, Stuttgart, v. I2, p. |-5, jan. |99|.

KESKINEN, K. L., KOMI, P. V. Intracycle variation in force, velocity and power as a measure of technique performance during front crawl swimming. In: BOUISSET, S.; MÉTRAL, S.; MONOD, H. (eds.). Abstr XIVth ISB Congress of Biomechanics, Paris, p. 676-677, 1993.

KOLMOGOROV, S. V.; DUPLISCHEVA, O. A. Active drag, useful mechanical power output and hydrodynamic force coefficient in different swimming strokes at maximal velocity. Journal of Biomechanics,Amsterdam, v. 25, p. 31।-318, 1992.

LAURENT JR, C. M. et al. Cross-validation of the 20- versus 30-s Wingate anaerobic test. European Journal of Applied Physiology, Heidelberg, v. I00, p. 645-65 I, 2007.

MORÉ, F. C.; CASTRO, F. A. S.; GUIMARÃES, A. C. S. Relação entre força aplicada por braçada e performance em nadadores e triatletas. In: XI CONGRESSO BRASILEIRO DE BIOMECÂNICA, 2005, João Pessoa. Anais... João Pessoa, 2005.

PAPOTI, M. et al. Padronização de um protocolo específico para determinação da aptidão anaeróbia de nadadores utilizando células de carga. Revista Portuguesa de Ciências do Desporto, Porto, v. 3, n. 3, p. 36-42, 2003. 
RISCH, O.; CASTRO, F. A. S. Desempenho em natação e pico de força em tethered swimming. In: XII CONGRESSO BRASILEIRO DE BIOMECÂNICA, São Pedro, 2007. Anais... São Pedro, 2007.

SWAINE, I. A.; DOYLE, G. Relationships between the mean arm-pulling and leg-kicking power output of semi-tethered and simulated front crawl swimming. In: KESKINEN, K.; KOMI, P.; HOLLANDER, A. (eds.). Biomechanics and medicine in swimming VIII. Jyväskylä: Department of Biology of Physical Activity, University of Jyväskylä, p. 363-368, 1999.

TAYLOR, S. et al. The effects of age, maturation and growth on tethered swimming performance. In: CHATARD, J. C. (ed.). Biomechanics and medicine in swimming IX. SaintÉtienne: Publications de l'Université de Saint-Étienne, p. 185-190, 2003.

TOUSSAINT, H. M.; BEEK, P. J. Biomechanics of competitive front crawl swimming. Sports Medicine, Indianapolis, v. 13, n. I, p. 8-24, 1992.

WHITEBREAD, M. T. et al. Post-exercise blood lactate decline after training in competitive cyclists and triathletes. Research Quartely for Exercise and Sport, Reston, v. 76, p. 238-242, feb. 2005.

YEATER, R. A. et al. Tethered swimming in the crawl, breast and back strokes and their relationship to competitive performance. Journal of Biomechanics, Amsterdam, v. 14, n. 8, p. 527-537, 1981.

Recebido: 18 jun. 2009 Aprovado: 25 out. 2009

Endereço para correspondência Flávio Antônio de Souza Castro ESEF-LAPEX R. Felizardo, 750 - Jardim Botânico Porto Alegre-RS CEP 90690-00 